\title{
Mechanisms and Biological Roles of Contact- Dependent Growth Inhibition Systems
}

\author{
Christopher S. Hayes ${ }^{1,2}$, Sanna Koskiniemi ${ }^{1}$, Zachary C. Ruhe ${ }^{1}$, Stephen J. Poole ${ }^{1}$, and \\ David A. Low ${ }^{1,2}$ \\ ${ }^{1}$ Department of Molecular, Cellular and Developmental Biology, University of California, Santa Barbara, \\ Santa Barbara, California 93106-9625 \\ ${ }^{2}$ Biomolecular Science and Engineering Program, University of California, Santa Barbara, Santa Barbara, \\ California 93106-9625 \\ Correspondence: low@lifesci.ucsb.edu
}

\begin{abstract}
Bacterial contact-dependent growth inhibition (CDI) is mediated by the CdiA/CdiB family of two-partner secretion proteins. $\mathrm{CDI}^{+}$cells bind to susceptible target bacteria and deliver a toxic effector domain derived from the carboxyl terminus of CdiA (CdiA-CT). More than 60 distinct CdiA-CT sequence types have been identified, and all CDI toxins characterized thus far display RNase, DNase, or pore-forming activities. CDI systems also encode Cdil immunity proteins, which specifically bind and inactivate cognate CdiA-CT toxins to prevent autoinhibition. CDI activity appears to be limited to target cells of the same species, suggesting that these systems play a role in competition between closely related bacteria. Recent work on the CDI system from uropathogenic Escherichia coli (UPEC 536) has revealed that its CdiA-CT toxin binds tightly to a cysteine biosynthetic enzyme (CysK) in the cytoplasm of target cells. The unanticipated complexity in the UPEC CDI pathway raises the possibility that these systems perform other functions in addition to growth inhibition. Finally, we propose that the phenomenon of CDI is more widespread than previously appreciated. Rhs (rearrangement hotspot) systems encode toxin-immunity pairs, some of which share significant sequence identity with CdiA-CT/Cdil proteins. A number of recent observations suggest that Rhs proteins mediate a distinct form of CDI.
\end{abstract}

$T^{\text {the }}$ he term "microbiome" was introduced to encompass microbes, their combined genomes, and their physiological interactions within a specific environment (Lederberg 2000). We have coevolved with our microbiome, and the microorganisms that colonize us play a significant role in determining health and resistance to disease (Dominguez-Bello and Blaser 2008; Benson et al. 2010; Ley 2010). The enor- mous diversity of bacterial species that reside within our intestines presents a daunting task if we wish to determine all the antagonistic and synergistic interactions they have with us and each other. Bacterial pathogenesis focuses on the relatively few species that cause adverse outcomes for the host, and has been explored to a much greater extent than bacterial ecology, which encompasses all interactions within the

Editors: Pascale Cossart and Stanley Maloy

Additional Perspectives on Bacterial Pathogenesis available at www.perspectivesinmedicine.org

Copyright (C) 2014 Cold Spring Harbor Laboratory Press; all rights reserved; doi: 10.1101/cshperspect.a010025

Cite this article as Cold Spring Harb Perspect Med 2014;4:a010025 
C.S. Hayes et al.

microbiome. However, this situation is likely to change as we realize that microbial ecology is central to our understanding of bacterial disease and the design of effective interventions. For example, Clostridium difficile infections are often precipitated by perturbations to the microbiome resulting from antibiotic treatment (Bakken et al. 2011). Moreover, antibiotic treatment can increase susceptibility to influenza virus through alterations in the microbiome and its interplay with the host immune system (Ichinohe et al. 2011). These examples illustrate the importance of developing therapies that target specific bacterial pathogens. One way to accomplish this goal is to understand the mechanisms by which bacteria interact and compete with one another. Significant progress has been made in understanding how bacteria communicate and compete via secreted chemicals and peptides (Keller and Surette 2006; Ng and Bassler 2009). Some secreted factors control the expression of virulence factors, and therefore blocking these signaling pathways provides a strategy for specific intervention (Chen et al. 2011). In contrast, comparatively little is known about bacterial communication mediated by direct cell-to-cell contact (Hayes et al. 2010; Konovalova and Sogaard-Andersen 2011; Pathak et al. 2012). Because bacteria in diverse environments grow on solid surfaces in biofilms, contact-dependent communication could play important roles that are distinct from the communication mediated by secreted signaling factors. This review focuses on contact-dependent growth inhibition (CDI), a mechanism that we discovered in 2005 (Aoki et al. 2005) and have recently shown plays a role in bacterial competition (Aoki et al. 2010).

\section{CDI}

The phenomenon of CDI was first described in Escherichia coli EC93, a wild-type isolate cultured from the intestinal tract of rats (Aoki et al. 2005). E. coli EC93 was found to profoundly inhibit the growth of most other E. coli strains in coculture experiments. Although enteric bacteria often produce soluble factors that kill related bacteria, the growth inhibition mediated by E. coli EC93 is unique in that it requires direct cell-to-cell contact with target cells. The CDI locus in E. coli EC93 is comprised of a threegene cluster ( $c$ diBAI), which is sufficient to confer the CDI inhibitor phenotype when introduced into laboratory strains of E. coli K-12 (Fig. 1). The $c d i B$ and $c d i A$ genes encode members of the two-partner secretion protein family (Jacob-Dubuisson et al. 2013). CdiB is a predicted outer membrane $\beta$-barrel protein that is required for the secretion and presentation of the CdiA exoprotein onto the cell surface. Like other two-partner secretion exoproteins, CdiA contains an amino-terminal transport domain followed by a hemagglutinin repeat region that is predicted to adopt a filamentous $\beta$-helical structure (Kajava et al. 2001). The CDI growth inhibitory activity is localized to the carboxy-terminal region of CdiA (CdiA-CT), and the cdiI gene product neutralizes the CdiA-CT toxin to protect E. coli EC93 cells from autoinhibition. The CdiI immunity protein is also sufficient to protect $\mathrm{CDI}^{-}$target cells from growth inhibition mediated by the E. coli EC93 CdiA-CT toxin. Thus, the E. coli EC93 CDI system encodes both toxin and immunity functions that are presumably used for growth competition.

\section{Systems Are Modular}

The proliferation of bacterial genome sequence information has revealed that CDI systems are widely distributed throughout the $\alpha-, \beta-$, and $\gamma$-proteobacteria and are commonly found in human, plant, and arthropod pathogens. Proteobacterial species contain zero to multiple CDI loci (Fig. 1). In Table 1, CdiA proteins present in a number of pathogenic bacterial species are annotated with accession numbers. CdiA proteins from $\gamma$-proteobacteria typically share sequence identity along most of the amino-terminal hemagglutinin repeat region, but the CdiA-CT sequences diverge abruptly after a common VENN peptide motif (Aoki et al. 2010; Poole et al. 2011). CdiA-CT sequences can also vary considerably between CDI systems from different strains of the same species. The predicted CdiI immunity proteins are also variable, indicating that CdiA-CT/CdiI pairs constitute a family of polymorphic toxin-immunity 


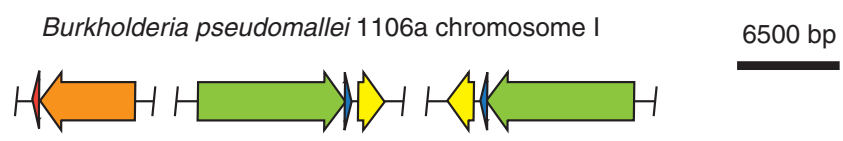

Burkholderia pseudomallei 1106a chromosome II
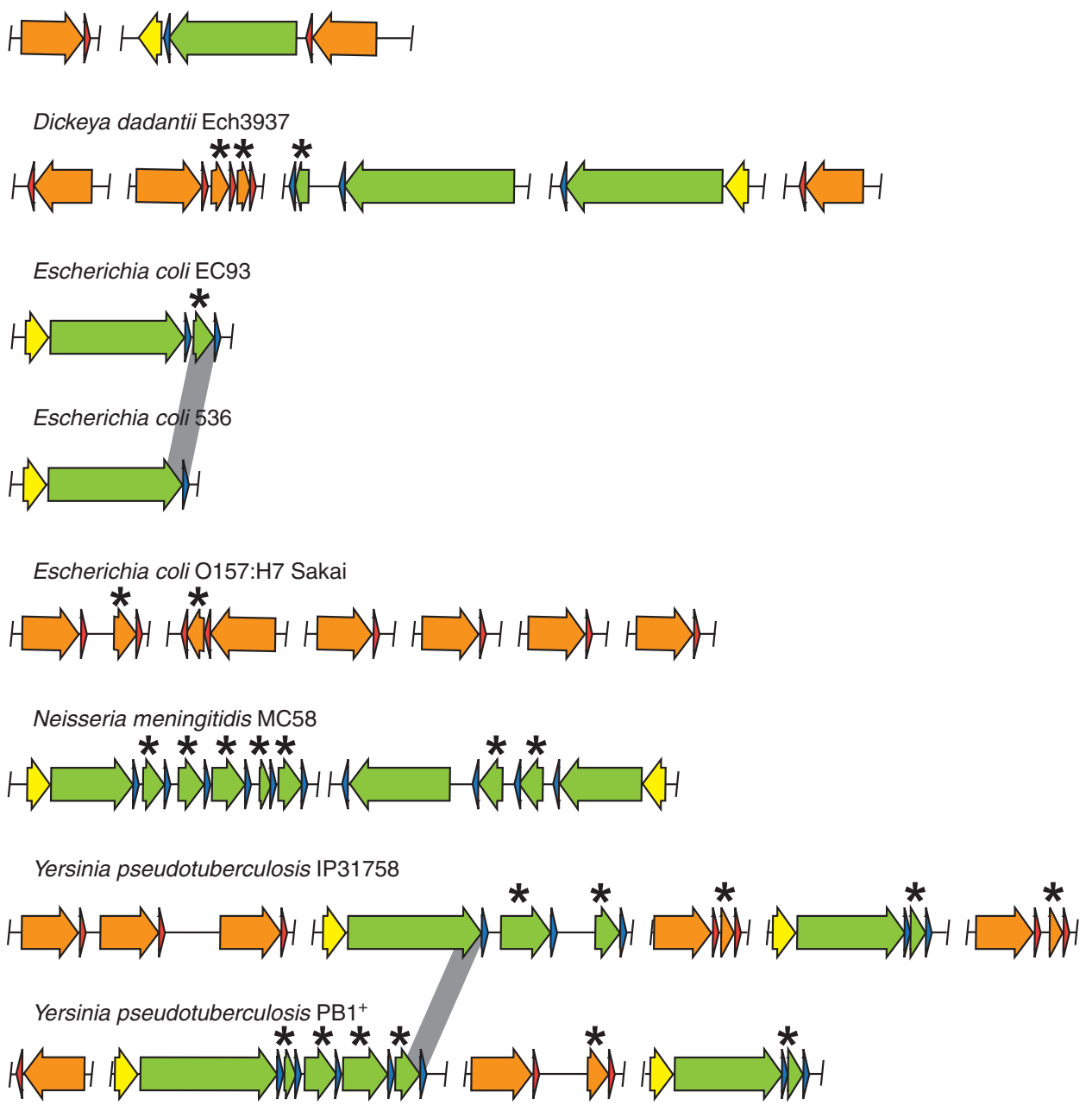

Figure 1. Organization of $c d i$ and $r h s$ genes in select bacterial pathogens. The $c d i$ and $r h s$ loci present in the chromosome(s) of the indicated bacterial pathogens are shown. Gaps between loci indicate that they are not closely linked. The order of gene regions and orientation of genes is as in the published genomic sequences, except for E. coli 536 and Yersinia pseudotuberculosis $\mathrm{PB}^{+}$, which have been reversed. Orphan cdiA-CTand rhs$C T$ regions are denoted by asterisks. Symbol key: $c d i A$, green arrows; $c d i B$, yellow arrows; $c d i I$, short blue arrows; $r h s$, orange arrows; and $r h s I$, short red arrows. Shaded regions show toxin-immunity modules that are found as an orphan in one strain and part of a full $C d i A$ gene in another. Selected pathogens illustrate different aspects of gene organization. Burkholderia pseudomallei 1106a, gene order $c d i A-I-B$ instead of the typical $c d i B-A-I$ gene order (chromosomes I and II) and rhs gene linkage to a CDI module (chromosome II); E. coli O157:H7 Sakai, rhs genes present but CDI modules absent; E. coli EC93, E. coli 536, and Neisseria meningitides MC58, CDI modules but rhs genes absent; N. meningitides MC58, multiple CDI modules with orphan cdiA-CT genes; B. pseudomallei 1106a, Dickeya dadantii Ech3937, Y. pseudotuberculosis IP31758, and Y. pseudotuberculosis $\mathrm{PB1}^{+}$, CDI modules together with Rhs modules; E. coli EC93, E. coli 536, Y. pseudotuberculosis IP31758, and Y. pseudotuberculosis $\mathrm{PB}^{+}$, exchange of toxin-immunity pairs between orphan $c d i A-C T$ and full $c d i A-C T$ genes. 
C.S. Hayes et al.

Table 1. CdiA and Rhs proteins encoded in select pathogens

\begin{tabular}{|c|c|c|}
\hline Species and strain & CdiA protein IDs & Rhs protein IDs \\
\hline Acinetobacter baumannii str. ACICU & ACC57223 (no orphans) & $\begin{array}{l}\text { ACC56430 (no orphans), } \\
\text { ACC56574 (no orphans) }\end{array}$ \\
\hline Bartonella grahamii as4aup & $\begin{array}{l}\text { YP_002971801 (no orphans), } \\
\text { YP_002972394 (no orphans), } \\
\text { YP_002972429 (no orphans), } \\
\text { YP_002972520 (no orphans); } \\
\text { two additional loci lack a Pfam } \\
\text { PF04829 domain }\end{array}$ & No rhs genes \\
\hline Bartonella tribocorum str. CIP 105476 & $\begin{array}{l}\text { CAK00851 (two orphans), } \\
\text { CAK00866 (four orphans), } \\
\text { CAK01426 (one orphan), } \\
\text { CAK01664 (two orphans) }\end{array}$ & No rhs genes \\
\hline $\begin{array}{l}\text { Burkholderia pseudomallei 1106a } \\
\text { chromosome I }\end{array}$ & $\begin{array}{l}\text { ABN92080 (no orphans), } \\
\text { ABN91683 (no orphans) }\end{array}$ & ABN90290 (no orphans) \\
\hline $\begin{array}{l}\text { Burkholderia pseudomallei 1106a } \\
\text { chromosome II }\end{array}$ & ABN92652 (no orphans) & $\begin{array}{l}\text { ABN92960 (no orphans), } \\
\text { ABN93053 (no orphans) }\end{array}$ \\
\hline Dickeya dadantii Ech586 & $\begin{array}{l}\text { ACZ76770 (one orphan), } \\
\text { ACZ78808 (three orphans) }\end{array}$ & $\begin{array}{l}\text { ACZ76163 (no orphan), } \\
\text { ACZ76342 (no orphans) }\end{array}$ \\
\hline Dickeya dadantii 3937 & $\begin{array}{l}\text { ADM98580 (one orphan), } \\
\text { ADM98660 (no orphans) }\end{array}$ & $\begin{array}{l}\text { ADM97066 (no orphans), } \\
\text { ADM97666 (two orphans), } \\
\text { ADM99131 (no orphans) }\end{array}$ \\
\hline Dickeya zeae Ech1591 & $\begin{array}{l}\text { ACT06763 (two orphans), } \\
\text { ACT06855 (no orphans) }\end{array}$ & $\begin{array}{l}\text { ACT05308 (no orphans), } \\
\text { ACT06027 (no orphans), } \\
\text { ACT06362 (one orphan), } \\
\text { ACT07636 (no orphans), } \\
\text { ACT07641 (no orphans, but } \\
\text { ACT07636 is immediately } \\
\text { downstream) }\end{array}$ \\
\hline Edwardsiella ictaluri 93-146 & $\begin{array}{l}\text { ACR70209 (one orphan), } \\
\text { ACR70465 (frameshift in cdiA; } \\
\text { five orphans) }\end{array}$ & No rhs genes \\
\hline Erwinia pyrifoliae Ep1/96 & CAX56670 (three orphans) & $\begin{array}{l}\text { CAX54630 (no orphans), } \\
\text { CAX54893 (one orphan), } \\
\text { CAX56922 (three orphans) }\end{array}$ \\
\hline Escherichia coli 536 & ABG72516 (no orphans) & No rhs genes \\
\hline Escherichia coli O157:H7 str. Sakai & No $c d i A$ genes & $\begin{array}{l}\text { BAB33660 (one orphan), } \\
\text { BAB34028 (one orphan), } \\
\text { BAB34152 (no orphans), } \\
\text { BAB35484 (no orphans), } \\
\text { BAB37893 (no orphans), } \\
\text { BAB38287 (no orphans) }\end{array}$ \\
\hline Escherichia coli CFT073 & AAN78830 (two orphans) & No rhs genes \\
\hline Escherichia coli UT189 & ABE10349 (one orphan) & No rhs genes \\
\hline Klebsiella pneumoniae str. 342 & ACI08381 (no orphans) & No rhs genes \\
\hline Neisseria meningitidis Z2491 & CAM07945 (three orphans) & No rhs genes \\
\hline Neisseria meningitidis MC58 & $\begin{array}{l}\text { AAF40927 (no orphans), } \\
\text { AAF40929 (five orphans), } \\
\text { AAF42109 (no orphans; linked to } \\
\text { AAF42119), AAF42119 (one orphan) }\end{array}$ & No rhs genes \\
\hline
\end{tabular}


Table 1. Continued

\begin{tabular}{|c|c|c|}
\hline Species and strain & CdiA protein IDs & Rhs protein IDs \\
\hline Pectobacterium carotovorum WPP163 & $\begin{array}{l}\text { ACX86275 (no orphans), } \\
\text { ACX88282 (no orphans) }\end{array}$ & $\begin{array}{r}\text { ACX86200 (seven orphans), } \\
\text { ACX86474 (no orphans), } \\
\text { ACX87399 (no orphans), } \\
\text { ACX88449 (one orphan) }\end{array}$ \\
\hline $\begin{array}{l}\text { Photorhabdus asymbiotica subsp. } \\
\text { asymbiotica ATCC } 43949\end{array}$ & $\begin{array}{l}\text { CAQ82538 (no } c d i B \text {; two orphans), } \\
\text { CAQ83255 (no orphans), } \\
\text { CAQ83577 (five orphans) }\end{array}$ & $\begin{array}{l}\text { CAQ85102 (no orphans), } \\
\text { CAQ83059 (no orphans), } \\
\text { CAQ85097 (no orphans) }\end{array}$ \\
\hline $\begin{array}{l}\text { Photorhabdus luminescens subsp. } \\
\text { laumondii }\end{array}$ & $\begin{array}{l}\text { NP_927589 (no orphans), } \\
\text { NP_927898 (no orphans), } \\
\text { NP_928461 (one orphan), } \\
\text { NP_928667 (no orphans), } \\
\text { NP_930296 (no orphans), } \\
\text { NP_930925 (one orphan) }\end{array}$ & NP_930352 (no orphans) \\
\hline $\begin{array}{l}\text { Yersinia pestis bv. Antiqua str } \\
\text { Antiqua }\end{array}$ & $\begin{array}{l}\text { ABG13952 (one orphan), } \\
\text { ABG15152 (no orphans) }\end{array}$ & $\begin{array}{l}\text { ABG15350 (no orphans), } \\
\text { ABG15382 (has an upstream } \\
\text { orphan) }\end{array}$ \\
\hline $\begin{array}{l}\text { Yersinia pseudotuberculosis serotype } \\
\text { O:3 str. YPIII }\end{array}$ & ACA67915 (one orphan) & $\begin{array}{l}\text { ACA66708 (ACA66715 just } \\
\text { downstream), } \\
\text { ACA66715 (no orphans), } \\
\text { ACA67067 (three orphans) }\end{array}$ \\
\hline
\end{tabular}

proteins. Together these observations suggest that CdiA proteins are modular, with the large amino-terminal $\beta$-helical "stick" region capable of delivering a variety of CdiA-CT toxins, each associated with a distinct immunity protein. To test this hypothesis, we generated $c d i A$ chimeras that encode the amino-terminal region of CdiA from uropathogenic E. coli 536 (UPEC 536) fused at the VENN motif to heterologous CdiA-CTs from Yersinia pestis CO92, E. coli EC93, and Dickeya dadantii 3937. The resulting chimeric CdiA proteins are functional in CDI against E. coli target cells (Aoki et al. 2010). Moreover, target cells are only protected if they express the CdiI immunity protein that corresponds to the grafted CdiA-CT, consistent with the specificity of cognate CdiA-CT/CdiI interactions. This modularity suggests that $c d i A$ genes may evolve rapidly by horizontal gene transfer and recombination to deploy novel toxins. This hypothesis is supported by metagenomic analysis indicating that $c d i A-C T / c d i I$ modules are actively exchanged between CDI systems (Poole et al. 2011).

\section{Orphan Toxin-Immunity Modules}

Many CDI systems contain tandem arrays of $c d i A-C T / c d i I$ toxin-immunity gene pairs downstream from the main $c$ diBAI gene cluster (Fig. 1, green arrows with asterisks). The $c d i A-C T$ sequences typically encode the conserved VENN peptide motif, but lack translation initiation signals. We have termed these gene pairs "orphan modules," because the cdiA-CT sequences appear to be displaced from full-length $c d i A$ genes. Orphan cdiA-CT/cdiI modules from E. coli EC93, D. dadantii 3937 and E. coli EC869 each encode a functional CdiA-CT toxin that is specifically neutralized by the corresponding orphan CdiI immunity protein (Poole et al. 2011). Thus, orphan $c d i A-C T / c d i I$ modules have the capacity to mediate CDI, but their deployment requires a mechanism to load orphan toxins onto full-length CdiA for export and assembly on the cell surface. There is evidence of orphan $c$ diA-CT/cdiI module translocation based on metagenomic analysis. For example, comparison of CDI loci from E. coli 
C.S. Hayes et al.

EC93/E. coli 536 or from Yersinia pseudotuberculosis IP31758/Y. pseudotuberculosis $\mathrm{PB} 1^{+}$indicates that an orphan $c d i A-C T / c d i I$ module in one strain is fused to the full-length $c d i A$ gene in another strain (Fig. 1) (Poole et al. 2011). Because CdiA-CTs are interchangeable, it is likely that the resulting CdiA chimera is functional and delivers the orphan CdiA-CT to neighboring cells. We propose that orphan $c d i A-C T / c d i I$ modules represent a reservoir of toxin diversity that confers a growth advantage to bacteria expressing them (S Koskiniemi, CS Hayes, and DA Low, unpubl.). Most orphan $c$ diA-CT fragments also encode nonvariable, CdiA "stick" sequences that are identical to the upstream full-length $c d i A$ gene. These direct repeat regions could facilitate homologous recombination, but such an event would delete the $c$ diI immunity gene and render the recombinants susceptible to growth inhibition by neighboring sibling cells. Alternatively, duplication of the CDI region could occur before recombination of an orphan $c d i A-C T /$ cdiI module onto one of the duplicated $c d i A$ genes. Under this scenario, the recombinant cell would express a novel CdiA protein loaded with an orphan CdiA-CT, yet would remain immune to the parental CDI system because it retains the main cdiI gene. Such recombinants could conceivably have a competitive advantage if the parental cells do not express sufficient orphan CdiI protein to confer immunity to the new chimeric CdiA protein.

\section{THE CDI PATHWAY}

Initial work on the CDI pathway has focused on the system deployed by E. coli EC93 (Aoki et al. 2005). A model for CDI is shown in Figure 2, which depicts the events known to occur following contact between a CDI ${ }^{+}$inhibitor cell and a target cell. These events are described below in detail.

\section{Binding to Target Cells}

Genetic selections for E. coli K-12 mutants that are resistant to the CDI system from $E$. coli isolate $\mathrm{EC} 93\left(\mathrm{CDI}^{\mathrm{EC} 93}\right)$ identified the outer membrane protein BamA (also known as YaT and
Omp85) as a possible CDI receptor on target cells (Aoki et al. 2008). A single transposon insertion just upstream of the bamA open reading frame was found to confer a $\mathrm{CDI}^{\mathrm{R}}$ phenotype, and mutants carrying this allele (termed bamA101) have $\sim 10$-fold less BamA on the cell surface compared with wild-type E. coli cells. BamA is a highly conserved, essential protein that is required for the assembly of all $\beta$-barrel proteins into the outer membrane (Bos et al. 2007; Knowles et al. 2009; Ricci and Silhavy 2012) and therefore the resistance conferred by bamA101 could be an indirect effect. To test whether BamA itself is the CDI receptor, target cells were preincubated with $\alpha$-BamA antibodies and then challenged with inhibitor cells expressing the $\mathrm{CDI}^{\mathrm{EC} 93}$ system. This antibody treatment protected target cells from growth inhibition, whereas treatment with preimmune serum or antibodies to the periplasmic POTRA3 domain of BamA showed no protective effect (Aoki et al. 2008). Moreover, expression of BamA lacking the POTRA-3 domain (BamA $\Delta \mathrm{P} 3)$ in the bamA101 background restored susceptibility to CDI. BamA $\Delta \mathrm{P} 3$ is stable and localizes to the outer membrane, but is not functional in $\beta$-barrel protein assembly. Thus, the biogenesis function of BamA, which involves interaction with other members of the $\beta$-barrel assembly machine (Ricci and Silhavy 2012), is not required for CDI. BamA is also required for the stable binding of $\mathrm{CDI}^{+}$inhibitor cells to target cells. Coculture of fluorescently labeled $\mathrm{CDI}^{+}$ inhibitor cells and target cells produces a distinct population of inhibitor-target cell aggregates that can be quantified by flow cytometry. The bamA101 mutation reduces target cell adhesion to inhibitor cells $\sim 10$-fold, and the interaction between $\mathrm{CDI}^{+}$cells and wild-type bamA ${ }^{+}$target cells can be blocked by $\alpha$-BamA antisera but not antibodies to the POTRA-3 domain (Aoki et al. 2008). Taken together, these results indicate that BamA is the target cell receptor for the $\mathrm{CDI}^{\mathrm{EC} 93}$ system.

Growth inhibition mediated by the E. coli EC93 CDI system is species restricted. Although E. coli EC93 inhibits most other E. coli strains, it has no effect on the growth of related $\gamma$-proteobacteria including Salmonella enterica sero- 


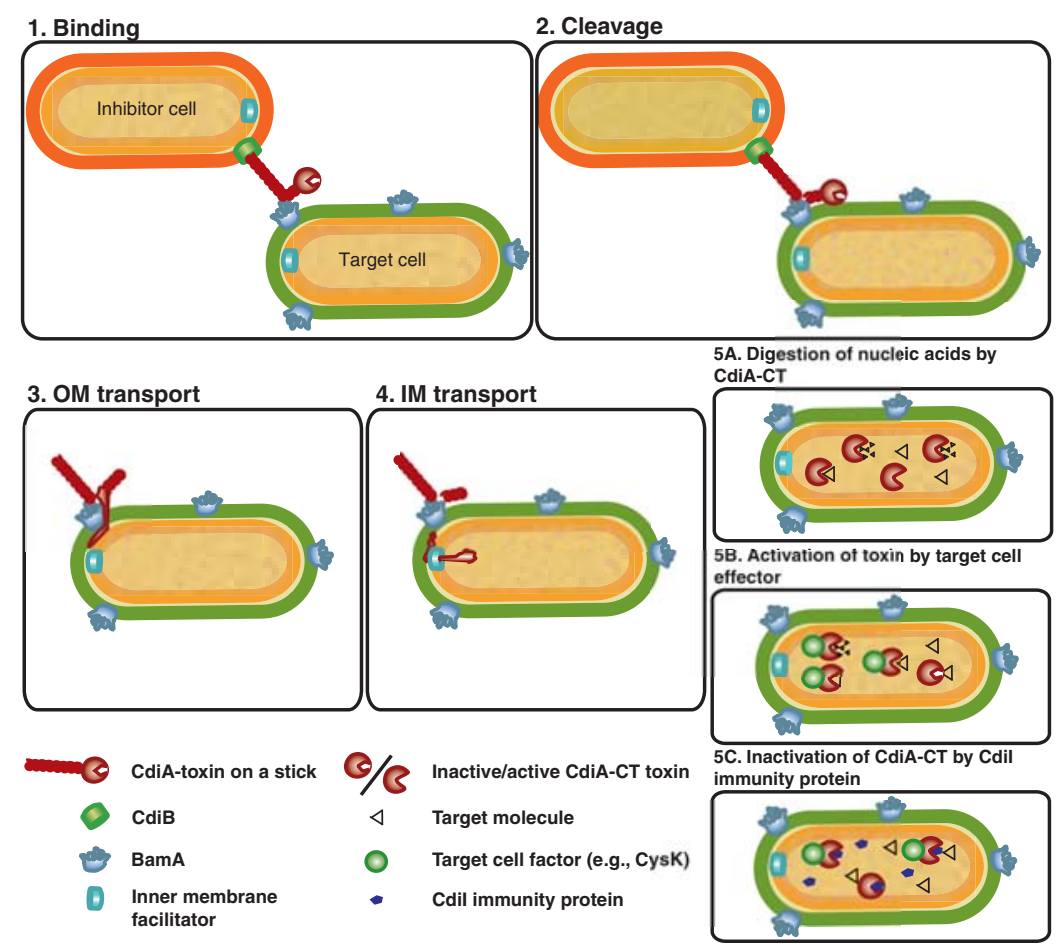

Figure 2. The CDI model. The major steps involved in CDI are depicted. $\mathrm{CDI}^{+}$inhibitor cells make contact with susceptible target cells through interactions between CdiA and the BamA receptor ( panel 1). The CdiA protein is then thought to undergo proteolytic cleavage ( panel 2) to liberate the CdiA-CT for transport through the target cell outer membrane (panel 3). Enzymatic CdiA-CT toxins are presumably transported through the inner membrane with the assistance of integral membrane proteins (panel 4). Once delivered into the target cell cytoplasm, nuclease toxins degrade DNA or RNA to inhibit cell growth. For the UPEC 536 CDI system, the CdiA-CT toxin requires activation by the CysK "permissive factor" (panels 5A and 5B). If target cells express the cognate CdiI immunity protein (e.g., isogenic $\mathrm{CDI}^{+}$siblings), then the CdiA-CT toxin is neutralized to prevent growth inhibition (panel 5C).

var Typhimurium, inhibitor-target cell aggregates, Citrobacter freundii, and Proteus mirabilis (Ruhe et al. 2013). These observations suggest that some step(s) in the $\mathrm{CDI}^{\mathrm{EC} 93}$ pathway is specific for E. coli target cells. This finding is somewhat unexpected because CDI systems are widely distributed across proteobacterial clades and appear to be horizontally transferred (Table 1). Multiple sequence alignment of BamA proteins from $\gamma$-proteobacteria show a high degree of sequence identity, but remarkably extracellular loops 4, 6, and 7 are highly variable in sequence and length (Fig. 3) (Noinaj et al. 2013). Through allelic exchange it was shown that bamA alleles from related species, such as Enterobacter cloacae, could compliment a bamA deletion in
E. coli while rendering these strains resistant to $\mathrm{CdiA}^{\mathrm{EC} 93}$. Additionally, binding of $\mathrm{CdiA}^{\mathrm{EC} 93}$ to BamAwas shown to require both loop 6 and loop 7 of E. coli BamA. These results suggest that the CDI target cell range results from a direct interaction between CdiA and extracellular loops 6 and 7 of E. coli BamA. This hypothesis was tested by grafting loops 6 and 7 from E. coli BamA onto BamA from Enterobacter cloacae. E. coli expressing this chimeric BamA bound to and were rendered susceptible to $\mathrm{CDI}^{\mathrm{EC} 93}$, supporting the hypothesis that intraspecies restriction of CDI is caused by cell receptor heterogeneity (Ruhe et al. 2013). Notably, the BamA proteins from Burkholderia species appear to lack extracellular loops entirely, suggesting that a different surface 
C.S. Hayes et al.

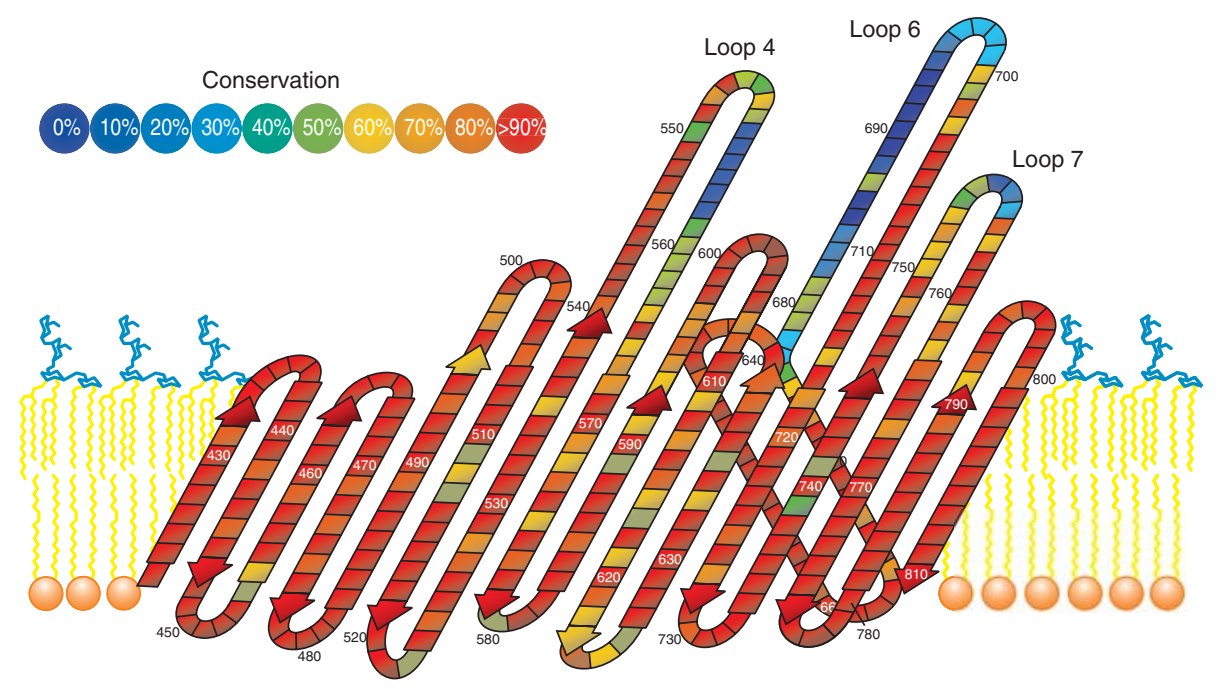

Figure 3. Conservation of BamA in the $\gamma$-proteobacteria. The predicted topology of BamA is presented with each residue color coded with a heat map indicating the degree of conservation (Ruhe et al. 2013). High and low degrees of conservation are shown as warm (red) and cool (blue) colors, respectively. Amino acid numbering is based on the preprocessed (including signal sequence) form of E. coli BamA. Conservation analysis was performed using the PRALINE multiple alignment tool (www.ibi.vu.nl/programs/pralinewww/) with an input of 40 different BamA sequences from $\gamma$-proteobacteria.

receptor may be exploited by the CDI systems from this group of $\beta$-proteobacteria.

\section{CdiA-CT Toxin Activities}

How do CDI systems inhibit the growth of target bacteria? Although there are approximately 60 distinct CdiA-CT/CdiI families, all characterized CDI toxins appear to either disrupt target cell membrane integrity or degrade cellular nucleic acids. During E. coli EC93-mediated $\mathrm{CDI}$, the target cell proton gradient is dissipated with an attendant decrease in respiration and ATP levels (Aoki et al. 2009). These findings suggest a CDI-dependent membrane pore that leaks ions. Presumably, the very hydrophobic $\mathrm{CdiI}^{\mathrm{EC} 83}$ immunity protein localizes to the inner membrane and prevents the assembly and/ or gating of the membrane pore. Other CdiACTs are nucleases that are specific for RNA or DNA. The CdiA-CT ${ }^{\mathrm{UPEC} 536}$ from UPEC 536 is a tRNase that cleaves in the anticodon loop of all tRNA isoacceptors, and CdiA-CT $T_{\text {II }}^{\text {Dd } 3937}$ from D. dadantii 3937 has $\mathrm{Mg}^{2+}$-dependent DNase activity (Aoki et al. 2010). Both of these CdiA-
CT nuclease activities are specifically blocked by the binding of cognate CdiI immunity proteins, suggesting that the nuclease activities are responsible for growth inhibition during CDI. The importance of nuclease activity has been established for the UPEC 536 CDI system. A $\mathrm{His}^{3193} \mathrm{Ala}$ missense change in the carboxy-terminal region of CdiA ${ }^{\text {UPEC536 }}$ ablates tRNase activity in vitro and completely blocks UPEC 536mediated CDI. The mutated CdiA-CT UPEC536 domain still binds to its cognate CdiI ${ }^{\mathrm{UPEC} 536}$ immunity protein, indicating that it retains its native tertiary structure. Moreover, the inactive variant is expressed on the inhibitor cell surface at the same level as wild-type $\mathrm{CdiA}^{\mathrm{UPEC} 536}$, demonstrating that the $\mathrm{His}^{3193}$ Ala mutation does not interfere with exoprotein biogenesis. Thus, although $\mathrm{CdiA}^{\mathrm{UPEC} 536}$ is a very large protein $(331 \mathrm{kDa})$ with the potential to carry multiple toxin activities, the tRNase activity contained within the carboxy-terminal $\sim 140$ residues accounts for all growth inhibition during CDI (Diner et al. 2012). Bioinformatic analyses indicate that a number of Cdil immunity proteins belong to the SUKH superfamily, 
which includes eukaryotic members such as Smil/Knr4 in yeast, which is involved in conferring resistance to a killer toxin (Zhang et al. 2011). By examining genes adjacent to those coding for SUKH family members a variety of putative nuclease and deaminase enzymes have been identified (Zhang et al. 2011).

\section{Identification of a Permissive Factor Required for CdiA-CT Activity}

Recent work has revealed unexpected complexity in the CDI pathway of UPEC 536 (Diner et al. 2012). As described above, the CDI ${ }^{\text {UPEC536 }}$ system delivers a CdiA-CTwith toxic tRNase activity into target cells. However, purified CdiA$\mathrm{CT}^{\mathrm{UPEC} 536}$ does not cleave tRNAs in vitro unless the reactions are supplemented with E. coli cell lysate, suggesting that an activating factor(s) is required for nuclease activity. Biochemical fractionation of E. coli lysates revealed that the CysK protein is necessary and sufficient to activate the $\mathrm{CdiA}^{-\mathrm{CT}}{ }^{\mathrm{UPEC} 536}$ tRNase in vitro. CysK is a biosynthetic enzyme that catalyzes the second step of L-cysteine synthesis from L-serine, but its enzymatic activity is not required to activate CdiA$\mathrm{CT}^{\mathrm{UPEC} 536}$. Instead, CysK binds to the carboxyterminal GYGI sequence of CdiA-CT ${ }^{\mathrm{UPEC} 536}$ to form a stable, active tRNase complex. Remarkably, the CdiA-CT ${ }^{\mathrm{UPEC} 536}$ GYGI motif mimics the GDGI peptide sequence found at the carboxyl terminus of CysE, which normally binds CysK to form the cysteine synthase complex in bacteria and plants. Thus, the CdiA-CT ${ }^{\text {UPEC536 }}$ toxin exploits an evolutionarily conserved proteinprotein interaction to promote nuclease activity. CysK is also required for CdiA-CT UPEC536 tRNase activity in vivo, and $\Delta c y s K$ mutants are completely resistant to cell-mediated $\mathrm{CDI}^{\mathrm{UPEC} 536}$ (Diner et al. 2012). Because target cells must express CysK to be inhibited by UPEC 536, we have designated CysK a "permissive factor." The requirement for a permissive factor is perplexing if CDI is viewed simply as a mechanism for bacterial competition, because target cells can readily acquire resistance through $c y s K$ mutations. Although there are likely to be fitness consequences for $c y s K$ mutations, these mutants are still prototrophic for cysteine owing to the pres- ence of CysM - an isoenzyme that catalyzes the same reaction as CysK, but does not activate the CdiA-CT ${ }^{\mathrm{UPEC} 536}$ toxin. One intriguing possibility is that CysK provides a mechanism for communication between UPEC 536 cells. There is no known exclusion mechanism in $\mathrm{CDI}$ and $\mathrm{CDI}^{+}$ cells appear to actively deliver CdiA-CTs to each other (manuscript in preparation). At face value, this exchange of toxins between immune cells appears futile, but perhaps the "inactive" CdiA-CT/CdiI complexes serve an unappreciated signaling function. The CdiA-CT ${ }^{\mathrm{UPEC} 536} \mathrm{can}$ bind to both CysK and the CdiI ${ }^{\mathrm{UPEC} 536}$ immunity protein simultaneously, suggesting that this ternary complex could affect cysteine metabolism or other pathways in UPEC 536 cells. We have recently found that the UPEC 536 CDI system modulates biofilm formation, although the molecular mechanism(s) underlying this phenotype has yet to be determined (Diner et al. 2012).

\section{Systems in Burkholderia Species}

The CDI systems found in Burkholderia, Cupriavidus, and Variovorax species are distinct from the systems found in other proteobacteria (Aoki et al. 2010; Anderson et al. 2012; Nikolakakis et al. 2012). These CDI systems are arranged in an alternative $C d i A I B$ gene order (Fig. 1) and lack the orphan $c d i A-C T / c d i I$ modules present in most other bacteria. B. pseudomallei serves as a convenient model to study Burkholderia CDI diversity because several clinical and environmental isolates have been sequenced, revealing significant genome heterogeneity between different strains. Our recent analysis of 85 assembled and unassembled B. pseudomallei genomes shows that almost every strain contains one to three predicted CDI systems (Nikolakakis et al. 2012). These systems fall into two classes based on the sequence of the amino-terminal hemagglutinin repeat region within CdiA proteins. The $B$. pseudomallei CDI systems can be further subdivided into 10 sequence types based on the polymorphic CdiA-CT region, which is demarcated by a conserved (Q/E)LYN peptide motif instead of the VENN sequence found in most other bacteria (Nikolakakis et al. 2012). Analysis 
C.S. Hayes et al.

of three different B. pseudomallei CdiA-CT/CdiI pairs confirmed that these proteins do indeed function as toxin-immunity pairs. Each toxin inhibits cell growth by cleaving tRNA at distinct positions within the anticodon loop, T-loop, or aminoacyl acceptor stem; and each tRNase activity is specifically blocked by its cognate CdiI immunity protein (Nikolakakis et al. 2012). The B. pseudomallei toxins are modular and can be exchanged between different Burkholderia CDI systems when fused at the $(\mathrm{Q} / \mathrm{E}) \mathrm{LYN}$ sequence within CdiA (Nikolakakis et al. 2012). However, B. pseudomallei CdiA-CTs are not functional when grafted onto E. coli CdiA proteins (Aoki et al. 2010; SK Aoki and DA Low, unpubl.), indicating that CDI pathways are significantly different in these two bacteria.

\section{Rhs Systems Are Functionally Similar to CDI}

CdiA proteins share a number of characteristics with the Rhs protein family. The rhs genes were first identified in E. coli by C.W. Hill and colleagues and were named rearrangement hotspots based on their role in chromosome duplications (Lin et al. 1984; Wang et al. 1998). Rhs proteins are widely distributed throughout the eubacteria, but their function is poorly understood. Like CdiA, Rhs proteins are large, ranging from $\sim 1500$ residues in Gram-negative bacteria to over 2000 residues in some Grampositive species. Rhs proteins also possess a central repeat region, although the characteristic YD peptide repeat of Rhs proteins is unrelated to the hemagglutinin repeats within CdiA. Moreover, Rhs proteins have variable carboxyterminal domains that are sharply demarcated by a conserved peptide motif (PxxxxDPxGL in the Enterobacteriaceae (Jackson et al. 2009). Remarkably, some Rhs and CdiA proteins share related carboxy-terminal sequences (Poole et al. 2011), indicating that the two protein families carry similar toxin domains. Moreover, CDI and Rhs systems have a similar genetic organization with several rhs loci containing one or more "silent cassettes" that resemble orphan $c d i A-C T / c d i I$ toxin-immunity modules (Hill et al. 1994; Poole et al. 2011). Like CDI systems, there are zero to multiple rhs loci in different bacterial strains (Fig. 1, Rhs $=$ orange and RhsI = red; Table 1 ). Taken together, the striking parallels between CDI and Rhs suggest that the two systems are functionally analogous.

Several independent lines of experimental evidence indicate that Rhs systems encode functional toxin-immunity protein pairs. Early work from Hill and colleagues showed that overexpression of the carboxy-terminal region of E. coli RhsA inhibited recovery from stationary phase (Vlazny and Hill 1995). This inhibitory activity appeared to be neutralized by coexpression of the downstream open reading frame (Vlazny and Hill 1995), suggesting this gene ( $y i b A$ ) encodes an antitoxin protein that blocks RhsA activity. A more recent study has shown that RhsA inhibits protein synthesis in E. coli, but the precise biochemical mechanism remains unknown (Aggarwal and Lee 2011). We recently showed that the Rhs systems in D. dadantii 3937 encode functional toxin-immunity pairs (Poole et al. 2011). The carboxyterminal regions of $D$. dadantii RhsA and RhsB proteins contain endonuclease NS (PF13930) and $\mathrm{HNH}$ endonuclease (PF01844) domains (respectively), and both proteins degrade cellular DNA when expressed in E. coli (Koskiniemi et al. 2013). These toxic activities are blocked specifically by small immunity proteins encoded immediately downstream from each $D$. dadantii 3937 rhs gene (Poole et al. 2011). These results, together with other reports indicating that Rhs proteins may be exported to the cell surface (Hill et al. 1994; McNulty et al. 2006) are consistent with the hypothesis that Rhs systems mediate a distinct form of CDI. We recently showed that RhsA and RhsB systems in the plant pathogen D. dadantii, and WapA, an Rhs homolog in Bacillus subtilis, mediate CDI and interbacterial competition (Koskiniemi et al. 2013). Previous work showed that Pseudomonas aeruginosa delivers an Rhs protein into eukaryotic cells where it acts as a virulence factor (Kung et al. 2012). These findings not only confirm that Rhs proteins are secreted toxins, but also define a new role for these systems in bacterial pathogenesis. 


\section{CONCLUDING REMARKS}

Although CDI was discovered nearly a decade ago, its importance in bacterial competition and cell-to-cell signaling is only beginning to be explored. The mechanisms of toxin delivery during CDI are not well understood, nor are the mechanisms responsible for the remarkable toxin-immunity diversity found throughout CDI systems. Insight into these fundamental features of CDI will significantly advance our understanding of interbacterial competition and contact-mediated signaling between bacterial cells. The orphan modules found in CDI and Rhs systems represent reservoirs of toxin-immunity protein diversity that can be potentially mobilized and deployed when needed, and rapidly lost under nonselective conditions. This concept is novel, but built on a foundation of research showing that gene duplications influence bacterial fitness (Reams et al. 2010) and that recombination provides a mechanism to rapidly alter the bacterial cell surface (Cahoon and Seifert 2009). Our recent data shows that Rhs constitutes a previously unrecognized CDI system (Koskiniemi et al. 2013). The function(s) of Rhs systems has been a mystery since their discovery in E. coli 30 years ago (Capage and Hill 1979). Rhs proteins are found throughout the eubacteria and eukaryotes (Minet and Chiquet-Ehrismann 2000), and therefore the elucidation of Rhs mechanisms and biological functions will have an impact on the study of a number of organisms.

\section{ACKNOWLEDGMENTS}

We thank Stephanie Aoki for unpublished data and members of the Hayes and Low laboratories for comments and helpful suggestions. This work was supported by grants GM078634 (to C.S.H.) and U54AI065359 (to C.S.H. and D.A.L.) from the National Institutes of Health, and grant 0642052 (to D.A.L.) from the National Science Foundation.

\section{REFERENCES}

Aggarwal K, Lee KH. 2011. Overexpression of cloned RhsA sequences perturbs the cellular translational machinery in Escherichia coli. J Bacteriol 193: 4869-4880.
Anderson MS, Garcia EC, Cotter PA. 2012. The Burkholderia $b c$ AIOB genes define unique classes of two-partner secretion and contact dependent growth inhibition systems. PLoS Genet 8: e1002877.

Aoki SK, Pamma R, Hernday AD, Bickham JE, Braaten BA, Low DA. 2005. Contact-dependent inhibition of growth in Escherichia coli. Science 309: 1245-1248.

Aoki SK, Malinverni JC, Jacoby K, Thomas B, Pamma R, Trinh BN, Remers S, Webb J, Braaten BA, Silhavy TJ, et al. 2008. Contact-dependent growth inhibition requires the essential outer membrane protein BamA (YaeT) as the receptor and the inner membrane transport protein AcrB. Mol Microbiol 70: 323-340.

Aoki SK, Webb JS, Braaten BA, Low DA. 2009. Contactdependent growth inhibition causes reversible metabolic downregulation in Escherichia coli. J Bacteriol 191: 17771786.

Aoki SK, Diner EJ, de Roodenbeke CT, Burgess BR, Poole SJ, Braaten BA, Jones AM, Webb JS, Hayes CS, Cotter PA, et al. 2010. A widespread family of polymorphic contactdependent toxin delivery systems in bacteria. Nature 468 : 439-442.

Bakken JS, Borody T, Brandt LJ, Brill JV, Demarco DC, Franzos MA, Kelly C, Khoruts A, Louie T, Martinelli LP, et al. 2011. Treating Clostridium difficile infection with fecal microbiota transplantation. Clin Gastroenterol Hepatol 9: 1044-1049.

Benson AK, Kelly SA, Legge R, Ma F, Low SJ, Kim J, Zhang M, Oh PL, Nehrenberg D, Hua K, et al. 2010. Individuality in gut microbiota composition is a complex polygenic trait shaped by multiple environmental and host genetic factors. Proc Natl Acad Sci 107: 18933-18938.

Bos MP, Robert V, Tommassen J. 2007. Biogenesis of the Gram-negative bacterial outer membrane. Ann Rev Microbiol 61: 191-214.

Cahoon LA, Seifert HS. 2009. An alternative DNA structure is necessary for pilin antigenic variation in Neisseria gonorrhoeae. Science 325: 764-767.

Capage M, Hill CW. 1979. Preferential unequal recombination in the glyS region of the Escherichia coli chromosome. J Mol Biol 127: 73-87.

Chen G, Swem LR, Swem DL, Stauff DL, O'Loughlin CT, Jeffrey PD, Bassler BL, Hughson FM. 2011. A strategy for antagonizing quorum sensing. Mol Cell 42: 199-209.

Diner EJ, Beck CM, Webb JS, Low DA, Hayes CS. 2012. Identification of a target cell permissive factor required for contact-dependent growth inhibition (CDI). Genes Dev 26: 515-525.

Dominguez-Bello MG, Blaser MJ. 2008. Do you have a probiotic in your future? Microbes Infect / Institut Pasteur 10: $1072-1076$.

Hayes CS, Aoki SK, Low DA. 2010. Bacterial contact-dependent delivery systems. Annu Rev Genet 44: 71-90.

Hill CW, Sandt CH, Vlazny DA. 1994. Rhs elements of Escherichia coli: A family of genetic composites each encoding a large mosaic protein. Mol Microbiol 12: 865-871.

Ichinohe T, Pang IK, Kumamoto Y, Peaper DR, Ho JH, Murray TS, Iwasaki A. 2011. Microbiota regulates immune defense against respiratory tract influenza A virus infection. Proc Natl Acad Sci 108: 5354-5359. 
C.S. Hayes et al.

Jackson AP, Thomas GH, Parkhill J, Thomson NR. 2009 Evolutionary diversification of an ancient gene family (rhs) through C-terminal displacement. BMC Genomics 10: 584 .

Jacob-Dubuisson F, Guerin J, Baelen S, Clantin B. 2013. Two-partner secretion: As simple as it sounds? Res Microbiol 164: 583-595.

Kajava AV, Cheng N, Cleaver R, Kessel M, Simon MN, Willery E, Jacob-Dubuisson F, Locht C, Steven AC. 2001. $\beta$-Helix model for the filamentous haemagglutinin adhesin of Bordetella pertussis and related bacterial secretory proteins. Mol Microbiol 42: 279-292.

Keller L, Surette MG. 2006. Communication in bacteria: An ecological and evolutionary perspective. Nat Rev Microbiol 4: 249-258.

Knowles TJ, Scott-Tucker A, Overduin M, Henderson IR. 2009. Membrane protein architects: The role of the BAM complex in outer membrane protein assembly. Nat Rev Microbiol 7: 206-214.

Konovalova A, Sogaard-Andersen L. 2011. Close encounters: Contact-dependent interactions in bacteria. $\mathrm{Mol} \mathrm{Mi-}$ crobiol 81: 297-301.

Koskiniemi S, Lamoureux JG, Nikolakakis KC, t'Kint de Roodenbeke C, Kaplan MD, Low DA, Hayes CS. 2013. Rhs proteins from diverse bacteria mediate intercellular competition. Proc Natl Acad Sci 110: 7032-7037.

Kung VL, Khare S, Stehlik C, Bacon EM, Hughes AJ, Hauser AR. 2012. An rhs gene of Pseudomonas aeruginosa encodes a virulence protein that activates the inflammasome. Proc Natl Acad Sci 109: 1275-1280.

Lederberg J. 2000. Infectious history. Science 288: 287-293.

Ley RE. 2010. Obesity and the human microbiome. Curr Opin Gastroenterol 26: 5-11.

Lin RJ, Capage M, Hill CW. 1984. A repetitive DNA sequence, rhs, responsible for duplications within the Escherichia coli K-12 chromosome. J Mol Biol 177: 1-18.

McNulty C, Thompson J, Barrett B, Lord L, Andersen C, Roberts IS. 2006. The cell surface expression of group 2 capsular polysaccharides in Escherichia coli: The role of KpsD, RhsA and a multi-protein complex at the pole of the cell. Mol Microbiol 59: 907-922.
Minet AD, Chiquet-Ehrismann R. 2000. Phylogenetic analysis of teneurin genes and comparison to the rearrangement hot spot elements of E. coli. Gene 257: 87-97.

$\mathrm{Ng}$ WL, Bassler BL. 2009. Bacterial quorum-sensing network architectures. Annu Rev Genet 43: 197-222.

Nikolakakis K, Amber S, Wilbur JS, Diner EJ, Aoki SK, Poole SJ, Tuanyok A, Keim PS, Peacock S, Hayes CS, et al. 2012. The toxin/immunity network of Burkholderia pseudomallei contact-dependent growth inhibition (CDI) systems. Mol Microbiol 84: 516-529.

Noinaj N, Kuszak AJ, Gumbart JC, Lukacik P, Chang H, Easley NC, Lithgow T, Buchanan SK. 2013. Structural insight into the biogenesis of $\beta$-barrel membrane proteins. Nature 501: 385-390.

Pathak DT, Wei X, Bucuvalas A, Haft DH, Gerloff DL, Wall D. 2012. Cell contact-dependent outer membrane exchange in myxobacteria: Genetic determinants and mechanism. PLoS Genet 8: e1002626.

Poole SJ, Diner EJ, Aoki SK, Braaten BA, T’Kint de Roodenbeke C, Low DA, Hayes CS. 2011. Identification of functional toxin/immunity genes linked to contact-dependent growth inhibition (CDI) and rearrangement hotspot (Rhs) systems. PLoS Genet 7: e1002217.

Reams AB, Kofoid E, Savageau M, Roth JR. 2010. Duplication frequency in a population of Salmonella enterica rapidly approaches steady state with or without recombination. Genetics 184: 1077-1094.

Ricci DP, Silhavy TJ. 2012. The Bam machine: A molecular cooper. Biochim Biophys Acta 1818: 1067-1084.

Ruhe ZC, Wallace AB, Low DA, Hayes CS. 2013. Receptor polymorphism restricts contact-dependent growth inhibition to members of the same species. mBio 4: e00480.

Vlazny DA, Hill CW. 1995. A stationary-phase-dependent viability block governed by two different polypeptides from the RhsA genetic element of Escherichia coli K-12. J Bacteriol 177: 2209-2213.

Wang YD, Zhao S, Hill CW. 1998. Rhs elements comprise three subfamilies which diverged prior to acquisition by Escherichia coli. J Bacteriol 180: 4102-4110.

Zhang D, Iyer LM, Aravind L. 2011. A novel immunity system for bacterial nucleic acid degrading toxins and its recruitment in various eukaryotic and DNA viral systems. Nucleic Acids Res 39: 4532-4552. 


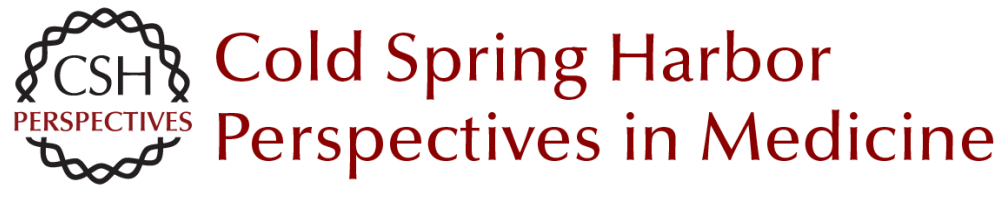

\section{Mechanisms and Biological Roles of Contact-Dependent Growth Inhibition Systems}

Christopher S. Hayes, Sanna Koskiniemi, Zachary C. Ruhe, Stephen J. Poole and David A. Low

Cold Spring Harb Perspect Med 2014; doi: 10.1101/cshperspect.a010025

Subject Collection Bacterial Pathogenesis

Therapeutic and Prophylactic Applications of

Bacteriophage Components in Modern Medicine Sankar Adhya, Carl R. Merril and Biswajit Biswas

Vaccines, Reverse Vaccinology, and Bacterial

Pathogenesis

Isabel Delany, Rino Rappuoli and Kate L. Seib

Helicobacter and Salmonella Persistent Infection

Strategies

Denise M. Monack

Echoes of a Distant Past: The cag Pathogenicity

Island of Helicobacter pylori

Nicola Pacchiani, Stefano Censini, Ludovico Buti, et al.

RNA-Mediated Regulation in Pathogenic Bacteria Isabelle Caldelari, Yanjie Chao, Pascale Romby, et al.

The Pneumococcus: Epidemiology, Microbiology, and Pathogenesis

Birgitta Henriques-Normark and Elaine I.

Tuomanen

Pathogenesis of Meningococcemia

Mathieu Coureuil, Olivier Join-Lambert, Hervé

Lécuyer, et al.

Chlamydial Intracellular Survival Strategies

Robert J. Bastidas, Cherilyn A. Elwell, Joanne N.

Engel, et al.
Mechanisms and Biological Roles of

Contact-Dependent Growth Inhibition Systems

Christopher S. Hayes, Sanna Koskiniemi, Zachary

C. Ruhe, et al.

A Genome-Wide Perspective of Human Diversity and Its Implications in Infectious Disease Jérémy Manry and Lluis Quintana-Murci

Host Specificity of Bacterial Pathogens Andreas Bäumler and Ferric C. Fang

The Inside Story of Shigella Invasion of Intestinal Epithelial Cells

Nathalie Carayol and Guy Tran Van Nhieu

Bartonella and Brucella--Weapons and Strategies for Stealth Attack

Houchaima Ben-Tekaya, Jean-Pierre Gorvel and Christoph Dehio

Concepts and Mechanisms: Crossing Host

Barriers

Kelly S. Doran, Anirban Banerjee, Olivier Disson, et al.

Genome Dynamics in Legionella: The Basis of

Versatility and Adaptation to Intracellular

Replication

Laura Gomez-Valero and Carmen Buchrieser

Mechanisms of Francisella tularensis Intracellular

Pathogenesis

Jean Celli and Thomas C. Zahrt

For additional articles in this collection, see http://perspectivesinmedicine.cshlp.org/cgi/collection/ 